SerpinA3N (30 nM) and a specific Elane inhibitor, Sivelestat $(100 \mu \mathrm{g} / \mathrm{ml})$ were used on cartilage explants treated by conditioned medium of macrophages pre-treated or not by IL-6 (CM-IL-6). Cartilage catabolism was determined by histology and matrix metalloproteinase MMP-3 production was evaluated by Western Blot and immunohistochemistry (IHC). Weekly intra-articular injections of Sivelestat $(1 \mathrm{mM})$ were performed in the DMM to determine the role of Elane in OA.

Results: $\triangle$ Serpina $3 \mathrm{n}^{\mathrm{Col} 2}$ mice had more severe OA lesions than control littermates 6 weeks after DMM, with greater cartilage damage (mean \pm SD OARSI score: $5.6 \pm 0.4$ vs $3.4 \pm 0.5, p=0.01)$, increased synovitis scores $(3.0 \pm 0.3$ vs $1.9 \pm 0.3, p=0.03)$ and bigger osteophytes $(7.2 \pm 0.8 \times 107$ vs $3.8 \pm 0.8 \times 107 \mu 3$, $\mathrm{p}=0.048)$. Conversely, WT mice treated with intra-articular injections of SerpinA3N 15nM exhibited less severe cartilage loss than mice treated with PBS after DMM (OARSI score: $2.1 \pm 0.4$ vs $3.9 \pm 0.5, p=0.02$ ). Elane mRNA expression was increased in macrophages upon IL-6 stimulation. In cartilage explants, CM-IL-6 activated cartilage catabolism and MMP-3 production, and effect that was blunted by SerpinA3N and Sivelestat. Finally, mice treated with intra-articular injections of Sivelestat had less severe cartilage damage than those treated with PBS after DMM (OARSI score: $3.3 \pm 0.47$ vs $5.8 \pm 0.53$, $\mathrm{p}=0.0046)$

Conclusion: SerpinA3N protects against experimental OA via the inhibition of Elane, a pro-catabolic serine protease produced by macrophages. This results highlight the crosstalk between cartilage and surrounding macrophages and open up new therapeutic perspectives.

Acknowledgments: This work has been supported by French Society of Rheumatology and ART Viggo association.

Disclosure of Interests: None declared

DOI: 10.1136/annrheumdis-2020-eular.4135

\section{OP0244 $\quad$ A PRECLINICAL TESTING TOOL: THE IN VITRO 3D FRACTURE GAP MODEL}

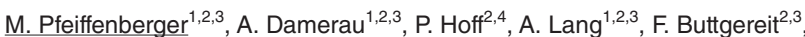
T. Gaber ${ }^{1,2,3}$. ${ }^{1}$ Universitätsmedizin Berlin, corporate member of Freie Universität Berlin, Humboldt-Universität zu Berlin, and Berlin Institute of Health, Berlin, Germany; ${ }^{2}$ Charité-Universitätsmedizin Berlin, Berlin, Germany; ${ }^{3}$ Department of Rheumatology and Clinical Immunology, Berlin, Germany; ${ }^{4}$ Endokrinologikum, Section Rheumatology, Berlin, Germany

Background: Approximately $10 \%$ of fractures lead to significant fracture healing disorders, with a tendency to further increase due to the aging population. Of note, especially immunosuppressed patients with ongoing inflammation show difficulties in the correct course of fracture healing leading to fracture healing disorders. Most notably, invading immune cells and secreted cytokines are considered to provide an inflammatory microenvironment within the fracture gap, primarily during the initial phase of fracture healing. Current research has the focus on small animal models, facing the problem of translation towards the human system. In order to improve the therapy of fracture healing disorders, we have developed a human cell-based in vitro model to mimic the initial phase of fracture healing adequately. This model will be used for the development of new therapeutic strategies.

Objectives: Our aim is to develop an in vitro 3D fracture gap model (FG model) which mimics the in vivo situation in order to provide a reliable preclinical test system for fracture healing disorders.

Methods: To assemble our FG model, we co-cultivated coagulated peripheral blood and primary human mesenchymal stromal cells (MSCs) mimicking the fracture hematoma (FH model) together with a scaffold-free bone-like construct mimicking the bony part of the fracture gap for $48 \mathrm{~h}$ under hypoxic conditions $(n=3)$, in order to reflect the in vivo situation after fracture most adequately. To analyze the impact of the bone-like construct on the in vitro $\mathrm{FH}$ model with regard to its osteogenic induction capacity, we cultivated the fracture gap models in either medium with or without osteogenic supplements. To analyze the impact of Deferoxamine (DFO, known to foster fracture healing) on the FG model, we further treated our FG models with either $250 \mu \mathrm{mol}$ DFO or left them untreated. After incubation and subsequent preparation of the fracture hematomas, we evaluated gene expression of osteogenic (RUNX2, SPP1), angiogenic (VEGF, IL8), inflammatory markers (IL6, IL8) and markers for the adaptation towards hypoxia ( $L D H A, P G K 1)$ as well as secretion of cytokines/chemokines using quantitative $\mathrm{PCR}$ and multiplex suspension assay, respectively.

Results: We found via histology that both the fracture hematoma model and the bone-like construct had close contact during the incubation, allowing the cells to interact with each other through direct cell-cell contact, signal molecules or metabolites. Additionally, we could show that the bone-like constructs induced the upregulation of osteogenic markers (RUNX2, SPP1) within the FH models irrespective of the supplementation of osteogenic supplements. Furthermore, we observed an upregulation of hypoxia-related, angiogenic and osteogenic markers (RUNX2, SPP1) under the influence of DFO, and the downregulation of inflammatory markers (IL6, IL8) as compared to the untreated control. The latter was also confirmed on protein level (e.g. IL-6 and IL-8). Within the bone-like constructs, we observed an upregulation of angiogenic markers (RNA-expression of VEGF, IL8), even more pronounced under the treatment of DFO.

Conclusion: In summary, our findings demonstrate that our established in vitro FG model provides all osteogenic cues to induce the initial bone healing process, which could be enhanced by the fracture-healing promoting substance DFO. Therefore, we conclude that our model is indeed able to mimic correctly the human fracture gap situation and is therefore suitable to study the influence and efficacy of potential therapeutics for the treatment of bone healing disorders in immunosuppressed patients with ongoing inflammation.

Disclosure of Interests: Moritz Pfeiffenberger: None declared, Alexandra Damerau: None declared, Paula Hoff: None declared, Annemarie Lang: None declared, Frank Buttgereit Grant/research support from: Amgen, BMS, Celgene Generic Assays, GSK, Hexal, Horizon, Lilly, medac, Mundipharma, Novartis, Pfizer, Roche, and Sanofi., Timo Gaber: None declared DOI: 10.1136/annrheumdis-2020-eular.3546

\section{OP0245 MICROBIOTA-INDUCED INTESTINAL BARRIER DYSFUNCTION PRECEDES THE ONSET OF ARTHRITIS AND ALLOWS THE SHUTTLING OF IMMUNE CELLS FROM THE GUT THE JOINTS}

M. Zaiss ${ }^{1}$, N. Taijc ${ }^{1}$, K. Sarter ${ }^{1}$, V. Azizov ${ }^{1}$, L. Bucci ${ }^{2}$, Y. Luo ${ }^{3}$, J. D. D. Cañete ${ }^{4}$, F. Ciccia ${ }^{5,6}$, G. Schett ${ }^{1} .{ }^{1}$ FAU Erlangen, Erlangen, Germany; ${ }^{2}$ Universita degli Studi della Campania, Napoli, Germany; ${ }^{3}$ Sichuan University, Chengdu, China;

${ }^{4}$ Servicio de Reumatolog ía, Barcelona, Spain; ${ }^{5}$ Universita degli Studi della Campania, Napoli, Italy; ${ }^{6}$ Department of Precision Medicine, Erlangen, Italy

Background: While it is known that microbial dysbiosis is associated with the onset of rheumatoid arthritis, mechanistic insights how it facilitates the development of arthritis remained largely elusive to date. It is especially interesting how microbial dysbiosis affects the transition from asymptomatic autoimmunity to arthritis. We speculated that a breakdown of intestinal barrier function caused by microbial dysbiosis allows immune cells to shuttle from the gut to the joints.

Objectives: To test whether intestinal barrier function is impaired before the onset of human RA and experimental arthritis and to seek for evidence that immune cells from the gut migrate to the joints.

Methods: In a longitudinal cohort of RA-at risk individuals markers of disturbed intestinal barrier function, such as zonulin, were analysed and linked to RA onset. Furthermore, new-onset RA patients were assessed for gut leakiness and their intestinal biopsies for the expression of tight junction proteins and immune cel infiltration. In the murine model of collagen-induced arthritis, sequential analysis of intestinal dysbiosis, intestinal barrier function and arthritis onset was carried out. Additionally, barrier function was assessed on intestinal organoids exposed to faecal supernatants from eu- and dysbiotic mice with and without inhibition of zonulin. Furthermore, three types of interventions restoring intestinal barrier function were carried out for testing their effects on the inhibition of arthritis onset. Finally, photo- converted cells from the gut were traced in the joints to tes for cellular trafficking from one to the other compartment.

Results: Zonulin, a potent regulator for intestinal tight junctions, was elevated in autoimmune mice and men before the onset of arthritis and predicted the onset of human RA. Intestinal barrier functions as well as epithelial tight junctions were decreased before the onset of experimental arthritis and at onset of human RA. In mice, induction of autoimmunity was followed by rapid intestinal dysbiosis followed by gut leakiness before arthritis started. Faecal supernatants of arthritic mice induce epithelial barrier dysfunction in intestinal organoids in zonulin dependent manner. Restoration of the intestinal barrier in the pre-phase of arthritis using butyrate, CB1R agonist or zonulin antagonist larazotide inhibited the development of arthritis. Finally, using photoconvertible mice, gut-borne immune cells were identified that homed to the joints when barrier function was impaired.

Conclusion: In summary, these data show the intestinal barrier dysfunction precedes the onset of RA and allows the trafficking of immune cells from the gut to the joints. Targeting of intestinal tight junction function may therefore allow preventing the onset of RA.

Acknowledgments: Funded by the DFG-FOR2886 PANDORA, DFG-CRC118, Staedtler foundation, Johannes und Frieda Marohn-Stiftung, Else Kröner-Fresenius foundation, Interdisciplinary Centre for Clinical Research, Erlangen (IZKF) BMBF-MASCARA and the IMI funded projectRTCure.

Disclosure of Interests: Mario Zaiss: None declared, Narges Taijc: None declared, Kerstin Sarter: None declared, Vugar Azizov: None declared, laura Bucci: None declared, Yubin Luo: None declared, Juan de Dios Cañete: None declared, francesco ciccia Grant/research support from: pfizer, novartis, roche, Consultant of: pfizer, novartis, lilly, abbvie, Speakers bureau: pfizer, novartis, lilly, abbvie, Georg Schett Speakers bureau: AbbVie, BMS, Celgene, Janssen, Eli Lilly, Novartis, Roche and UCB

DOI: 10.1136/annrheumdis-2020-eular.4251 